\title{
Permutation groups of prime degree, a quick proof of Burnside's theorem
}

\author{
Peter Müller
}

July 29, 2019

\begin{abstract}
A transitive permutation group of prime degree is doubly transitive or solvable. We give a direct proof of this theorem by Burnside which uses neither S-ring type arguments, nor representation theory.
\end{abstract}

In this note $\mathbb{F}_{p}$ is the field with $p$ elements, with $p$ a prime. The following proposition proves Burnside's theorem in a few lines.

Proposition 1. Let $U$ be a non-empty, proper subset of $\mathbb{F}_{p} \backslash\{0\}$. Let $\pi$ be a permutation of $\mathbb{F}_{p}$ such that $i-j \in U$ for $i, j \in \mathbb{F}_{p}$ implies $\pi(i)-\pi(j) \in U$. Then there are $a, b \in \mathbb{F}_{p}$ such that $\pi(i)=a i+b$ for all $i \in \mathbb{F}_{p}$.

In Sch08 Schur gives a proof of this proposition in two steps. First he uses a precursor of his S-ring technique to show that if $1 \in U$, then $U$ is a subgroup of $\mathbb{F}_{p} \backslash\{0\}$. In the second step he shows that $\pi$ is linear. In this note we show that a small modification of his second step makes the first step unnecessary. See the remarks at the end for further comments.

Proof of Burnside's theorem. Let $G$ be a transitive permutation group on $p$ elements. As $p$ divides the order of $G$, there is an element $\tau \in G$ of order $p$. Assume that $G$ acts on $\mathbb{F}_{p}$, with $\tau(i)=i+1$ for all $i \in \mathbb{F}_{p}$. Suppose that $G$ is not doubly transitive. So $G$ has at least two orbits on the pairs $(i, j)$ with $i \neq j$. On the other hand, $\tau$ permutes cyclically the pairs $(i, j)$ with constant difference, so there is a non-empty proper subset $U$ of $\mathbb{F}_{p} \backslash\{0\}$ such that $\pi(i)-\pi(j) \in U$ for all $\pi \in G$ and $i, j$ with $i-j \in U$. By the proposition, $G$ is a subgroup of the group of permutations $i \mapsto a i+b$ with $a \in \mathbb{F}_{p} \backslash\{0\}, b \in \mathbb{F}_{p}$. In particular, $G$ is solvable. 
Proof of the proposition. By an iterated application of $\pi$ we see that $i-j \in U$ if and only if $\pi(i)-\pi(j) \in U$. In particular, replacing $U$ by its complement in $\mathbb{F}_{p} \backslash\{0\}$ preserves the assumption. Therefore we may and do assume $|U| \leq \frac{p-1}{2}$.

Fix $i \in \mathbb{F}_{p}$. For $u \in U$ we have $(i+u)-i \in U$, hence $\pi(i+u)-\pi(i) \in U$. As $\pi$ is a permutation, the elements $\pi(i+u)-\pi(i)$ are different for different $u$. Thus $\{\pi(i+u)-\pi(i) \mid u \in U\}=U$, hence $\{\pi(i+u) \mid u \in U\}=\{\pi(i)+u \mid u \in$ $U\}$. In particular, for $w \in \mathbb{N}$ we obtain

$$
\sum_{u \in U} \pi(i+u)^{w}=\sum_{u \in U}(\pi(i)+u)^{w}
$$

Let $f(X) \in \mathbb{F}_{p}[X]$ be the polynomial of degree $n \leq p-1$ with $f(i)=\pi(i)$ for all $i \in \mathbb{F}_{p}$. Suppose $w n \leq p-1$. Then $\sum_{u \in U} f(X+u)^{w}-\sum_{u \in U}(f(X)+u)^{w}$ is a polynomial of degree $<p$ which vanishes identically on $\mathbb{F}_{p}$, thus

$$
\sum_{u \in U} f(X+u)^{w}-\sum_{u \in U}(f(X)+u)^{w}=0
$$

Setting $S(k)=\sum_{u \in U} u^{k}$, we obtain

$$
\sum_{u \in U}\left(f(X+u)^{w}-f(X)^{w}\right)=\sum_{k \geq 1}\left(\begin{array}{l}
w \\
k
\end{array}\right) S(k) f(X)^{w-k}
$$

Note that $f(X)^{w}$ is a polynomial of degree $n w$, so $X^{n w}$ is an $\mathbb{F}_{p^{-}}$linear combination of the derivatives of $f(X)^{w}$. Thus we obtain

$$
\sum_{u \in U}\left((X+u)^{n w}-X^{n w}\right)=\sum_{k \geq 1} S(k) g_{w-k}(X)
$$

where $g_{\ell}(X)$ is a polynomial of degree at most $\ell n$.

Let $r \geq 1$ be minimal with $S(r) \neq 0$. Then the degree of the right handside is at most $n(w-r)$.

Suppose that $r \leq n w$. Then the coefficient of $X^{n w-r}$ on the left handside is (up to a nonzero factor) $S(r)$. Since $S(r) \neq 0$, we must have $n w-r \leq$ $n(w-r)$, so $n=1$, and we are done.

It remains to consider $r-1 \geq n w$. Suppose we have chosen $w$ maximal with $n w \leq p-1$. Then $p-1<n(w+1) \leq 2 n w \leq 2(r-1)$, so $r>(p+1) / 2$.

This shows $S(k)=0$ for $k=1,2, \ldots,(p-1) / 2$. Therefore $|U| \geq(p+1) / 2$ (for instance because the van der Monde matrix of $U$ is not singular; or 
because the first $(p-1) / 2$ elementary symmetric functions of $U$ vanish, so a polynomial with zero set $U$ has degree $\geq(p+1) / 2)$. This contradicts $|U| \leq(p-1) / 2$.

Remark. Our proof is similar to the final step in Schur's proof in [Sch08]. However, the main part of his proof consists in showing that if $1 \in U$, then $U$ is a subgroup of $\mathbb{F}_{p} \backslash\{0\}$. Thus if $1 \leq k \leq w<|U|$, then $S(k)=0$, so $\sum_{u \in U} f(X+u)^{w}=|U| f(X)^{w}$, which produces a contradiction similarly as above. See also [DM96, 3.5] for a modern version of this proof.

In [DKM92] the authors give an S-ring argument to show that $U$ is a group. From there they however proceed with geometric arguments, and use facts about lacunary polynomials to conclude that $\pi$ is a linear function.

Burnside's original proof uses complex character theory, see [Bur11].

The certainly most elegant proof is due to Wielandt, who studies the ring of $G$-invariant functions from $\mathbb{F}_{p}$ to $\mathbb{F}_{p}$. See [Wie94, pages 273-296], [HB82, $X I I, \S 10]$. A very concise and streamlined version of Wielandt's proof is contained in [LMT93, 6.7].

\section{References}

[Bur11] W. Burnside, Theory of Groups of Finite Order, Cambridge Univ. Press, 2nd edn. (1911).

[DKM92] A. W. M. Dress, M. H. Klin, M. E. Muzichuk, On p-configurations with few slopes in the affine plane over $\mathbf{F}_{p}$ and a theorem of $W$. Burnside's, Bayreuth. Math. Schr. (1992), 40, 7-19.

[DM96] J. D. Dixon, B. Mortimer, Permutation Groups, Springer-Verlag, New York (1996).

[HB82] B. Huppert, N. Blackburn, Finite Groups III, Springer-Verlag, Berlin Heidelberg (1982).

[LMT93] R. Lidl, G. L. Mullen, G. Turnwald, Dickson Polynomials, vol. 65 of Pitman Monographs and Surveys in Pure and Applied Mathematics, Longman, Essex (1993).

[Sch08] I. Schur, Neuer Beweis eines Satzes von W. Burnside, Jahresbericht der Deutsch. Math.-Ver. (1908), 17, 171-176. 
[Wie94] H. Wielandt, Mathematische Werke/Mathematical works. Vol. 1, Walter de Gruyter \& Co., Berlin (1994).

IWR, Universität Heidelberg, Im Neuenheimer Feld 368, 69120 Heidelberg, Germany

E-mail: Peter.Mueller@iwr.uni-heidelberg.de 